\title{
Research on Satellite Signal Detection Scheme
}

\author{
ZHANG BIAO ${ }^{1, a}$, SHEN TAO ${ }^{2, b,{ }^{*}}$ and WANG DI ${ }^{3, c}$ \\ ${ }^{1}$ No. 2, Tongxin Road, Hongqing Town, Baqiao District, Xi'an, Shaanxi Province, China \\ 2 No. 2, Tongxin Road, Hongqing Town, Baqiao District, Xi'an, Shaanxi Province, China \\ ${ }^{3}$ No. 2, Tongxin Road, Hongqing Town, Baqiao District, Xi'an, Shaanxi Province, China \\ azhangbiao199403@163.com, b594147555@qq.com, c1312703428@qq.com \\ ${ }^{*}$ Corresponding author
}

Keywords: Satellite signal, Beacon signals, Satellite TV signal, Communication carrier signal.

\begin{abstract}
This paper introduces the characteristics of common source of the satellite signal detection module. At present, the satellite signal detection module usually detects satellite beacon signals, which has high costs and weak anti-interference, and is not suitable for use in a variety of complex environments. In order to overcome the shortcomings in the current mobile satellite communication signal detection scheme, a multi-source signal detection scheme is proposed. This scheme can use different detection sources according to different working environments and working conditions to ensure the smoothness of mobile satellite communication links. This solution is robust, adaptable to various environments, simple in structure, low in cost, and especially suitable for low-cost development of mobile satellite communication equipment.
\end{abstract}

\section{Introduction}

With the increase of satellite resources, mobile satellite communications has become the mainstream of future satellite communications [1]. It has the advantages of long communication distance, wide coverage area, wide communication frequency bandwidth, large transmission capacity, high communication quality, and flexible communication links [2,3]. At present, satellite resources in the sky can cover any corner of the earth. In other words, as long as there are satellite communication equipment terminals, they can quickly access the Internet and communicate with the outside world at any corner of the earth. According to the kind of receiving terminal, satellite communications can be divided into fixed satellite communications and mobile satellite communications. Mobile satellite communications can play an outstanding role in deserts, islands, and emergency rescue and other special environments. It is the mainstream of future development of satellite communications. The representative equipment of satellite communication is also known as "SOTM", that is satellite on the move. This paper focuses on the scheme used by the satellite signal detection module in the "SOTM" system $[4,5]$.

Downlink signals of satellite links usually include satellite beacon signals, satellite television signals, and communication carrier signals [6]. These three signals have different characteristics. The program can fully utilize the characteristics of different satellite signals according to different working environments of the satellite signal detection module.

\subsection{Satellite Beacon Signals}

The beacon signal is a kind of special signal that identifies the satellite. It is usually used by satellite ground monitoring stations to search, track, monitor, and control satellites. From the launch of the satellite, the beacon signal will continue to work until the end of the life of the satellite. Usually there is a beacon signal in each polarization direction. Beacon signals are usually distributed at both ends of the downlink signal band, and $\mathrm{Ku}$-band beacons are usually located near the $12250 \mathrm{MHz}$ and 12750 $\mathrm{MHz}$ frequencies. Figure 1 shows the use of a spectrum analyzer to measure the beacon signals of 
Asia NO.V. Since the ground receiver contains $11300 \mathrm{MHz}$ local oscillators, the detected beacon signal frequency is $1449.7 \mathrm{MHz}$ (Vertically polarized beacon frequency is $12749.75 \mathrm{MHz}$ ) [7].

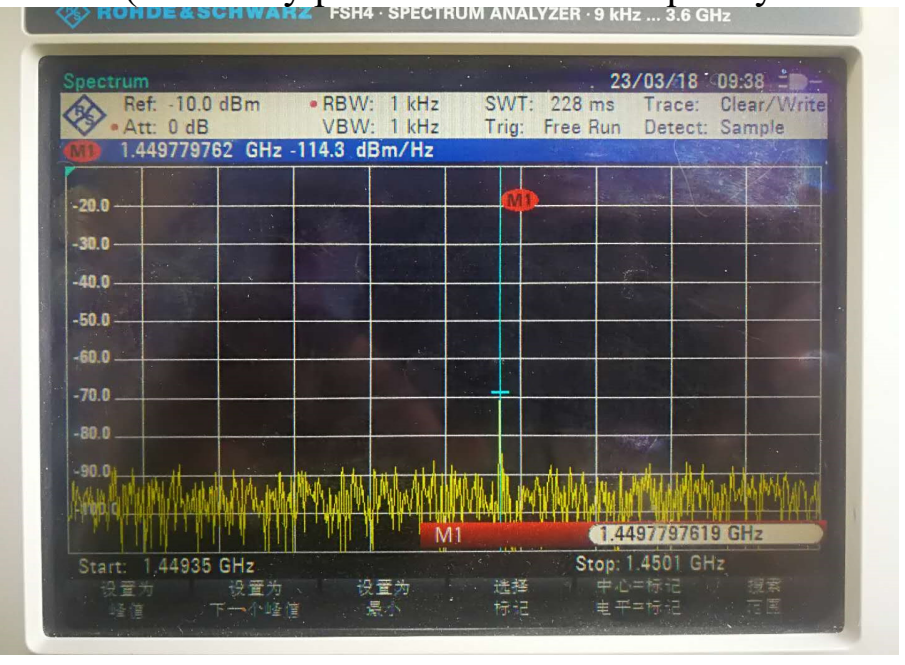

Fig. 1. Beacon signal spectrogram

\subsection{Satellite television signals}

Satellite TV signals (DVB-S, DVB-S2) have the characteristics of wide frequency band and high intensity. In addition to the audio and video contents, the data stream output after receiving, tuning and demodulating also includes many auxiliary data information. Data information can complete the satellite identification. The data stream is transmitted in packet format. The header identification byte is $0 x 47$. The NIT (Network Information Table) in the data stream provides information about the physical network. The NIT carries network identifier (network ID, NID) information. Each has a unique identifier [8,9]. Since the NID in the NIT is unique, the satellite can be identified by extracting the NID in the data stream. Some of the satellite network identifiers (NIDs) are shown in Table.1.

Table 1. Parts of the satellite NID

\begin{tabular}{|c|c|c|}
\hline Satelite & Direction & $\begin{array}{c}\text { NID } \\
(\text { Frequncy }(\mathrm{GHz}))\end{array}$ \\
\hline Zhongwei No.1 & $87.5^{\circ} \mathrm{E}$ & $7000(3.869)$ \\
\hline Zhongxin No.1 & $88^{\circ} \mathrm{E}$ & $1121(3.632)$ \\
\hline Asia NO.3S & $105.5^{\circ} \mathrm{E}$ & $100(12.316)$ \\
\hline Xinnuo NO.1 & $110.5^{\circ} \mathrm{E}$ & $65(12.320)$ \\
\hline Asia NO.4 & $122^{\circ} \mathrm{E}$ & $8888(12.320)$ \\
\hline Yatai NO.6 & $134^{\circ} \mathrm{E}$ & $8022(12.302)$ \\
\hline Yatai NO.5 & $138^{\circ} \mathrm{E}$ & $41029(12.720)$ \\
\hline
\end{tabular}

To identify satellites with satellite television signals requires demodulation and decoding of satellite television signals. This requires high hardware requirements, high costs, and is not economical. However, in the phase of satellite tracking, the real-time adjustment of the antenna attitude in real-time can be adjusted according to the variation of satellite TV signal strength, which can ensure the real-time openness of the communication link. Such a scheme is simple in structure and low in cost.

\subsection{Communication carrier signals}

The bandwidth of the communication carrier signal is between the beacon signal and the satellite television signal, which is typically a few megahertz bandwidth. Different satellites often use different modulation and coding methods. Satellites can be identified according to different modulation and coding methods. 


\section{Satellite detection and identification scheme}

In the course of work, the "SOTM" system will undergo initial capture, tracking, shadowing, and then recapture phases. The state transition is shown in Figure 2

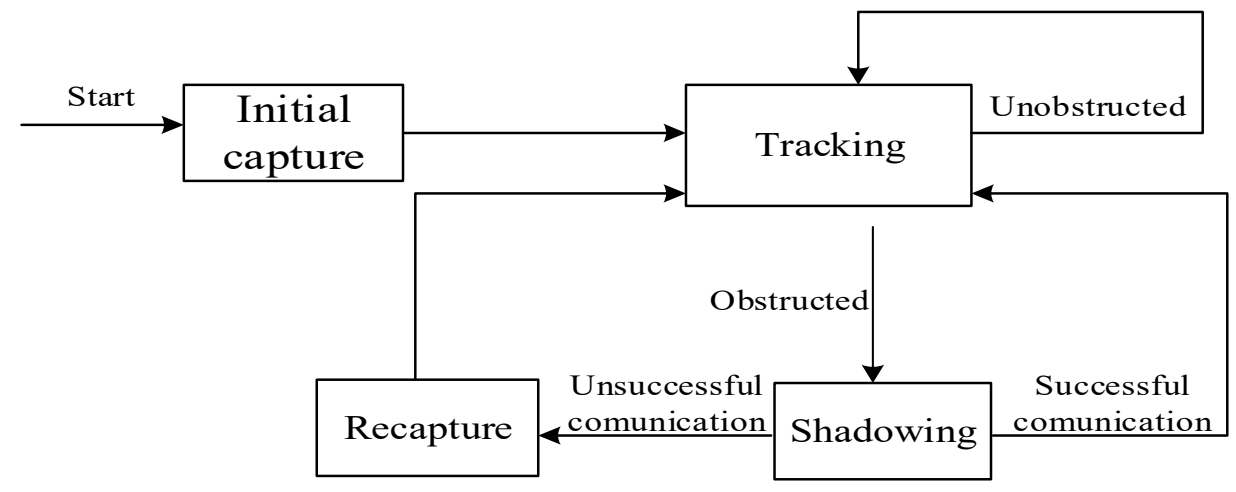

Fig. 2. State transition diagram

This paper synthetically utilizes the characteristics of narrow band and uniqueness of beacon signal, wide frequency band and high intensity of satellite TV signal, and proposes a satellite identification tracking scheme for narrow-band identification satellites and wide-band tracking satellites. It is used for initial acquisition and reacquisition in the "SOTM" system. During the stage of searching for the target satellite, it cannot be determined whether the detected signal is a signal forwarded by the target satellite. By detecting whether the beacon signal is received or not, it is determined whether the "SOTM" system is aimed at the target satellite. In the tracking phase, the carrier posture changes little. The "SOTM" antenna beam has been aligned with the approximate airspace where the target satellite is located. By detecting changes in the strength of the satellite television signal, according to the principle of maximum signal strength, the antenna pointing is fine-tuned so that the antenna beam is accurately aligned with the satellite at all times to ensure the communication chain. The scheme does not require demodulation and decoding of satellite television signals. It only uses the signal strength of satellite television signals as a feedback signal to instruct the measurement and control system to adjust the antenna beam, which reduces the complexity of the system. The structure is simple, the cost is low, the response speed is fast, and it is easy to implement. Hardware structure scheme diagram shown in Figure 3

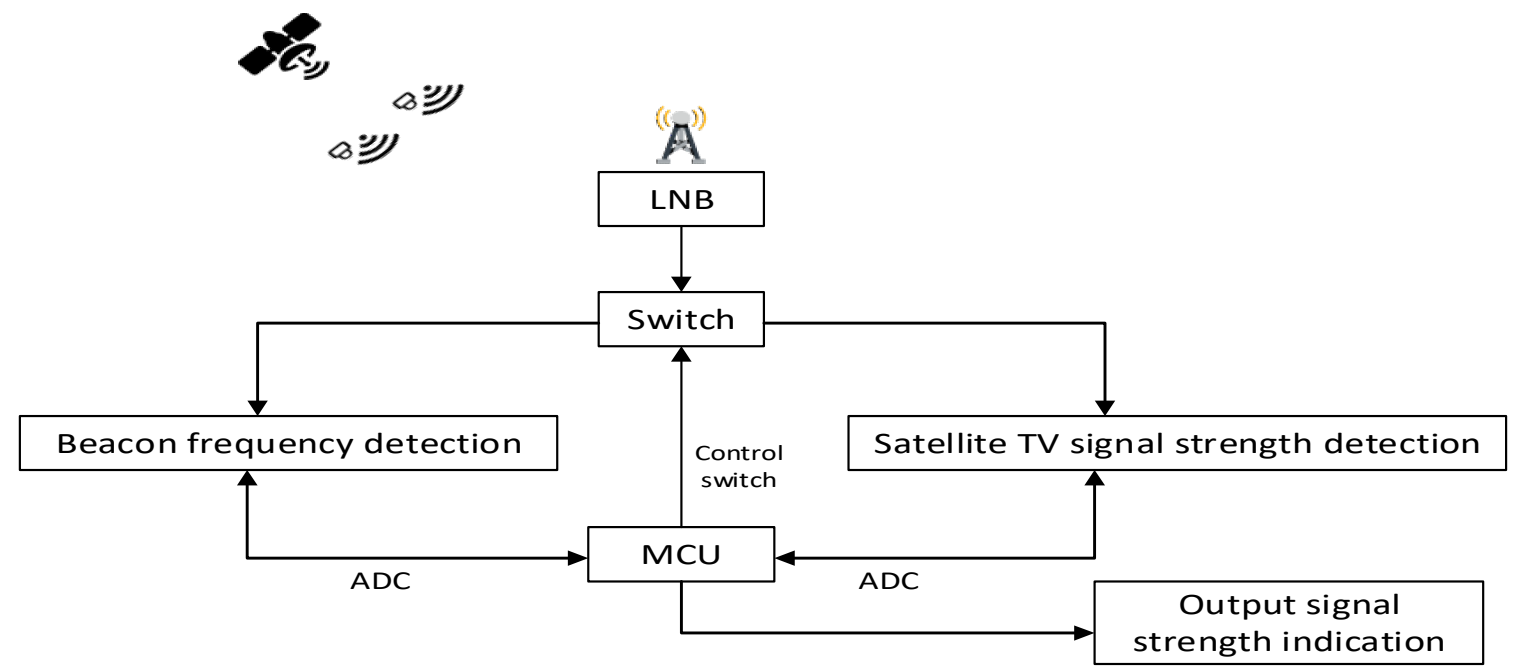

Fig. 3. System structure

\section{Acknowledgement}

This research was financially supported by the research on Key Technologies of Low-cost 
Measurement and Control of Satellite Communication of China (Grant NO. 61179005)

\section{References}

[1] Zhang Feng-gan. Measurement and Control Techniques for SatCom on-the-Move[D]. The second artillery engineering university, 2009.

[2] Li Chang-yong, Run Ying-min. Design and Realization of Satellite Identification system based on Single Chip Micyoco [J]. Micro computer information, 2008, 24(17):95-96.

[3] Hao Lu-yao,Zhao Jian-xun,Su Gang. The technology of stabilization and tracking in satellite communication in motion [J]. Radar and confrontation, 2006(2):48-51.

[4] Zhang Bin, Zhang Feng-gan, Ren Jia-Wei. Signal Derection in Satellite Receiver Based on I2C Bus. The Chinese cable TV[J], 2009(2):149-152.

[5] Wu Zong-wei. Research on Key Techniques of Low Cost M\&C System for SOTM[D]. The second artillery engineering university,2009.

[6] He Cai-yu.The Application of STV0299B in Satellite Digital TV Receiver[J]. The Chinese cable TV,

[7] Chinese find starnet[DB/OL]. http://www.cnsat.com

[8] Zhang Dong-chen. Military communication: The nervous system of information war[M]. Beijing: National defence industry press, 2008.

[9] Li Xue-jun. "SOTM" Satellite Signal Detection System Based on DVB-S[J]. Computer Disc Software and Applications, 2014, 17(09): 275-276. 